\title{
EFEITO DE EXTRATOS DE ARISTOLOCHIA LAGESIANA (ARISTOLOCHIACEAE) SOBRE A LAGARTA-DA-SOJA, ANTICARSIA GEMMATALIS (LEPIDOPTERA: NOCTUIDAE)
}

\section{Vieira ${ }^{1 *}$, I. De Pascoli² ${ }^{2}$ S.A. De Bortoli ${ }^{3}$, L.M.X. Lopes $^{2}$}

${ }^{1}$ Universidade de São Paulo, Faculdade de Filosofia, Ciências e Letras, Departamento de Biologia, Av. dos Bandeirantes, 3900, CEP 14040-901, Ribeirão Preto, SP, Brasil. E-mail: vieira@aluno.ffclrp.usp.br

\section{RESUMO}

\begin{abstract}
Vários relatos deatividades biológicas podem ser encontrados nas espécies de plantas pertencentes ao gênero Aristolochia (Aristolochiaceae), cujas plantas estão distribuídas em todo o território nacional. Visando a obtenção de mais informações sobre essas plantas, avaliou-sea ação deextratos de Aristolochia lagesiana Ule. var. intermedia Hoehne sobre Anticarsia gemmatalis Hüebner, 1818(Lepidoptera: Noctuidae). O experimento foi conduzido no Laboratório de Biologia e Criação de insetos da FCAV/ UNESP Jaboticabal e no Laboratório de Química Orgânica do IQ/ UNESP - Araraquara. A toxidade relativa dos extratos foi determinada através da aplicação tópica de uma gota de $1 \mu \mathrm{Ldesolução,} \mathrm{decadaextrato,}$ quefoiaplicada no mesotórax delagartas deterceiro instar, utilizando-se um aparelho microaplicador. As plantas foram extraídas em acetona, que foi evaporada antes da aplicação e, para isto, os extratos foram re-solubilizados em etanol para obtenção das seguintes dosagens: 6,0; 9,6; 13,2; 16,8; 20,4 e 22,0 $\mathrm{mg} / \mu \mathrm{L}$. Os extratos de $A$. lagesiana alongam a duração do período larval, reduzem o peso de pupas e também diminuem a viabilidade larval de $A$. gemmatalis .
\end{abstract}

PALAVRAS-CHAVE: Planta inseticida, efeitos deletérios, extratos acetônicos.

\section{ABSTRACT}

EFFECT OF EXTRACTS OF ARISTOLOCHIA LAGESIANA (ARISTOLOCHIACEAE) ON VELVETBEAN CATERPILLARS. Various reports have shown that species of the genus Aristolochia, whose plants are distributed throughout Brazilian territory, can produce biological effects. The objective of the present study was to evaluate the action of extracts of Aristolochia lagesianaUle. var. intermedia Hoehne (Aristolochiaceae) on the velvetbean caterpillar. The bioassays were conducted at the Biology and Rearing Insect Laboratory of the FCAV/UNESP in Jaboticabal, state of São Paulo, SP, Brazil, and at the Chemistry Organic Laboratory of the IQ/UNESP in Araraquara, SP. The relative toxicity of the extracts was evaluated through the topical application of $1 \mu \mathrm{L}$ of extract solution on the mesothorax of third-instar Anticarsia gemmatalis larvae, using a microsyringe, connected to a microapplicator apparatus. Plants were extracted in acetone, which was completely evaporated before the application. For applying on insects, extracts were diluted in ethanol to obtain the following dosages: 6.0, 9.6, 13.2, 16.8, 20.4 and $22.0 \mathrm{mg} / \mu \mathrm{L}$. Extracts of $A$. lagesiana increased the duration of the larval phase, decreased the pupae weight and also the larval viability.

KEY WORDS: Insecticide plant, deleterious effects, acetone extracts.

\section{INTRODUÇÃO}

As pesquisas com plantas inseticidas são realizadascom o intuito de se descobrir novas moléculas que permitam a obtenção de novos produtos sintéticos e, também, a obtenção de inseticidas botânicos naturais para o uso direto no controle de pragas (Manejo Integrado de Pragas). Existem várias maneiras de se utilizar as plantas inseticidas, sendo o seu emprego mais comum na forma de pó seco, óleo e extratos aquosos ou orgânicos (metanólico, etanólico, acetônico, butanólico, clorofórmico, hexânico etc.). Os pós e extratos aquosos, que são de fácil obtenção e aplicação, constituem-se na melhor opção para o agricultor de baixa renda, que pode não dispor de recursos técnicos e econômicos para aquisição e aplicação dos produtos sintéticos (VENDRAMIM,CASTIGLIONI, 2000).

\footnotetext{
${ }^{2}$ Universidade Estadual Paulista, Instituto de Química, Departamento de Química Orgânica, Araraquara, SP, Brasil. ${ }^{3}$ Universidade Estadual Paulista, Faculdade de Ciências Agrárias, Departamento de Fitossanidade, Jaboticabal, SP, Brasil.
} 
Na década de 50, MARANHão (1954) relacionou cerca de 2.000 plantas inseticidas (distribuídas em 170 famílias) com atividade tóxica para diversos insetos. De acordo com esse autor, os inseticidas comerciais de origem vegetal eram obtidos, principalmente, de cinco famílias botânicas: Solanaceae, Leguminosae, Chenopodiaceae e Liliaceae, das quais se extraíam, respectivamente, a nicotina, piretro, timbó, heléboro e anabasina. GRAINGE; AHMED (1988) catalogaram 2.400 espécies de plantas com propriedades úteis no controle de insetos, além de listarem cerca de 800 pragas controladas poressas plantaseainda, 100 plantas com outras substâncias químicas reportadas no controle de doenças e nematóides parasitas do homem e de outros animais. SCHUMUTTERER (1988) citou as famílias Meliaceae, Asteraceae, Labiaceae, Aristolochiaceae e Annonaceae como principais fontes de princípios ativos inseticidas.

As espécies da família Aristolochiaceae possuem composição química muito diversificada de metabólitos secundários (ácidos graxos, terpenóides, lignóides, flavonóides e alcalóides) (Lopes et al., 2001), sendo que cerca de 670 compostos diferentes foram isolados de 192 espécies(Lopes et al., 2001), destacando-se os ácidos aristolóquicos, substâncias de ocorrência restrita às espécies do gênero Aristolochia (Venkadasubramanian; David, 1999; Sime et al., 2000; Wu et al., 2000; Lopes et al., 2001).

Os ácidos aristolóquicos apresentam atividade quimio-esterilizante e antialimentar em insetos, além de atividade contraceptiva, estimulação gástrica, redução do edema provocado pelo veneno de cobra, além de ação anti-inflamatória sobre o homem e/ou outros animais (Lopes et al., 2001).

Nas espécies de Aristolochia, há relatos de atividade inseticida e repelente, tendo como exemplo Aristolochia clematis e Aristolochia grandiflora que são usadas como repelentes de moscas e gafanhotos (SECOY; Sмітн, 1983). Aristolochia bracteata apresenta atividade inseticida para os mosquitosAnophelesarabinensis, Culex quinquefasciatus e Aedes aegypti (ZARroug et al., 1988). A atividade inseticida é confirmada também nos extratos metanólico e diclorometânico de Aristolochia triangularis para as larvas de A. aegypti, cujo adulto (fêmea) é o vetor da dengue hemorrágica (CiCCIA et al., 2000).

O presente trabalho teve como objetivo avaliar o efeito deletério dos extratos de Aristolochia lagesiana sobre alguns aspectos biológicos da lagarta-da-soja, A. gemmatalis.

\section{MATERIAL E MÉTODOS}

Criação e obtenção de Anticarsia gemmatalis -Os adultos de $A$. gemmatalis foram coletados no campo e conduzidos aolaboratório, onde foram acondicionados em gaiolas dePVC $(30 \mathrm{~cm}$ dealtura $20 \mathrm{~cm}$ de diâmetro), com seu interior revestido com papel sulfite, servindo como substrato para oviposição, sendo mantidos em sala climatizada (temperatura: $27 \pm 2^{\circ} \mathrm{C}$, umidade relativa: $80 \pm 10 \%$ e fotofase: 14 horas) (GAZzon et al.,1981).

Após o início da oviposição, a cada 2 dias foi realizada a coleta de posturas, que foram transferidas para placas de Petri de $10 \mathrm{~cm}$ de diâmetro, contendo dieta artificial (CAMPO etal., 1985), onde as lagartas eclodiam permanecendo até completar seu desenvolvimento, sendo mantidas em câmara climatizada tipo B.O.D, sob as mesmas condições dos adultos.

Coleta e identificação do material vegetal - As amostras vegetais foram coletadas em Ituiutaba, MG. Omaterial botânicofoiidentificadopeloDr. Condorcet Aranha, da Secretaria do Meio Ambiente da Prefeitura de Joinville, Joinville, SC.

Obtenção dos extratos de Aristolochialagesiana-Os extratos de A.lagesiana foram preparados no Laboratório de Produtos Naturais do Departamento de Química Orgânica do Instituto de Química, UNESP, Araraquara, SP. As diferentes partes do material vegetal, secas e moídas, foram submetidas à extração com acetona. As amostras testadas foram os extratos brutos obtidos.

Bioensaio de aplicação tópica - Foi realizado no Laboratório de Biologia e Criação de Insetos (LBCI) da Faculdade de Ciências Agrárias e Veterinárias da Universidade Estadual Paulista, Campus de Jaboticabal, $\mathrm{SP}$, sendo a aplicação tópica dos extratos feita em lagartas de terceiroínstar de A. gemmatalis. Emcadainsetofoi aplicado $1 \mathrm{~mL}$ desolução, no mesotórax entreosegundo e o terceiro par de pernas, sendo este tamanho de gota escolhido por assegurar uma cobertura eficiente, sem que ocorresse perda do produto por escorrimento. Empregou-se uma micros-seringa Hamilton de $100 \mathrm{~mL}$ acoplada a um aparelho microaplicador Burkard 900-x (Burkard Manufacturing Co. Ltda). Dos extratos acetônicos defolhas, caule/raiz (aquecido)ecaule/raiz (a frio) deA.lagesiana foram aplicad as, respectivamente, as concentrações de 6,0; 9,6;13,2; 16,8; 20,4 e 22,0 mg/ inseto, diluídas em etanol. Após a aplicação, as lagartas foram colocadas em placas de Petri forradas com papel filtro, contendo dieta artificial, sendo mantidas em sala climatizada do LBCI. Os tratamentos-controle foram realizados comaplicação do solvente puroeágua(solução Tween-20) sobre os insetos.

Análise estatística - $O$ delineamento experimental utilizado foi o inteiramente casualizado, com 10 tratamentos e 3 repetições, sendo cada repetição composta por 10 insetos. A avaliação dos efeitos deletérios dos extratos de A. lagesiana sobre os insetos foi realizada acompanhando os seguintes parâmetros biológicos: duração do período larval e pupal, viabilidade larval e pupal e peso de pupas. Os dados obtidos foram analisados pelo teste Tukey a $5 \%$ de probabilidade, utilizando o software Estat. 


\section{RESULTADOS E DISCUSSÃO}

Efeito dos extratos de Aristolochia lagesiana sobre Anticarsia gemmatalis

Duração do período larval - Analisando-se o período larval de A. gemmatalis foi verificado que houve aumento significativo na sua duração um nos diferentes tratamentos em relação à testemunha (Tabela 1). Embora estes não tenham diferido entre si, houve tendência de maior valor no tratamento "Caule/Raiz (aquecido)" , na dose de 13,2 $\mu \mathrm{g} /$ inseto (17,63 dias), caracterizando um alongamento de 3,5 dias do período larval. Estes resultados demonstram que houve um efeito adverso na biologia do inseto, que necessita de um maior número de dias para completar sua fase larval. NAscimentoet al. (2003) também observaram o alongamento do período larval de $A$. gemmatalis quando foram pulverizadas sobre estes insetos substâncias puras isoladas do extrato acetônico e etanólico de Aristolochia pubescens. Este fato foi comentado por RoEL (2001) como sendo um dos possíveis efeitos deletérios da ação de extratos de plantas com potencial inseticida sobre insetos.

Tabela 1 - Duração do período larval (dias) de A. gemmatalis submetida à aplicação tópica do extrato acetônico das diferentes partes de A. lagesiana (Aristolochiaceae).

\begin{tabular}{|c|c|c|c|c|c|c|c|}
\hline \multirow[t]{2}{*}{ Dose $\left(\mu \mathrm{g} . \mu \mathrm{L}^{-1}\right)$} & \multicolumn{7}{|c|}{ Período larval (dias) $)^{1}$} \\
\hline & (n) & Caule/Raiz (Aquecido) & (n) & Caule/Raiz (A frio) & $(\mathrm{n})$ & Folha & DMS \\
\hline Controle 1 & 30 & $14,13 \mathrm{bA}$ & 30 & $14,13 \mathrm{cA}$ & 30 & $14,13 \mathrm{bA}$ & - \\
\hline Controle 2 & 30 & $14,63 \mathrm{bA}$ & 30 & $14,63 \mathrm{cA}$ & 30 & $14,63 \mathrm{bA}$ & - \\
\hline 6,0 & 30 & $17,10 \mathrm{aA}$ & 23 & $16,93 \mathrm{abA}$ & 22 & $16,73 a A$ & 0,24 \\
\hline 9,6 & 23 & $16,52 \mathrm{aA}$ & 25 & $16,89 \mathrm{abA}$ & 24 & $16,70 \mathrm{aA}$ & 0,17 \\
\hline 13,2 & 20 & $17,05 \mathrm{aA}$ & 16 & $17,63 \mathrm{aA}$ & 28 & $17,09 a \mathrm{~A}$ & 0,12 \\
\hline 16,8 & 28 & $16,82 \mathrm{aA}$ & 27 & $16,55 \mathrm{bA}$ & 18 & $16,90 \mathrm{aA}$ & 0,13 \\
\hline 20,4 & 27 & $16,30 \mathrm{aA}$ & 19 & $17,42 \mathrm{abA}$ & 20 & $17,04 \mathrm{aA}$ & 0,15 \\
\hline 22,0 & 21 & $16,53 \mathrm{aA}$ & 22 & $17,29 \mathrm{abA}$ & 27 & $17,09 \mathrm{aA}$ & 0,19 \\
\hline DMS & 0,86 & 0,99 & 0,78 & - & & & \\
\hline C.V.(\%) & 6,77 & 7,55 & 6,13 & - & & & \\
\hline
\end{tabular}

${ }^{1}$ Médias seguidas pela mesma letra minúscula, na mesma coluna, e maiúscula, na mesma linha, não diferem significativamente entre si pelo teste de Tukey $(\mathrm{P}<0,05)$; $(\mathrm{n})$ : número de insetos usados no teste; Controle 1: Tween $20(0,1 \%)$; Controle 2: Etanol puro.

Tabela 2 - Duração período pupal (dias) de A.gemmatalis submetida à aplicação tópica do extrato acetônico das diferentes partes de A. lagesiana (Aristolochiaceae).

\begin{tabular}{|c|c|c|c|c|c|c|c|}
\hline \multirow[t]{2}{*}{ Dose $\left(\mu \mathrm{g} . \mu \mathrm{L}^{-1}\right)$} & \multicolumn{7}{|c|}{ Período pupal (dias) ${ }^{1}$} \\
\hline & (n) & Caule/Raiz (Aquecido) & (n) & Caule/Raiz (A frio) & (n) & Folha & DMS \\
\hline Controle 1 & 28 & $8,25 \mathrm{aA}$ & 28 & $8,25 \mathrm{aA}$ & 28 & $8,25 \mathrm{abA}$ & - \\
\hline Controle 2 & 28 & $8,34 \mathrm{aA}$ & 28 & $8,34 \mathrm{aA}$ & 28 & $8,34 \mathrm{abA}$ & - \\
\hline 6,0 & 20 & $7,91 \mathrm{aA}$ & 20 & $7,87 \mathrm{abA}$ & 20 & $8,46 \mathrm{aA}$ & 0,49 \\
\hline 9,6 & 16 & $7,94 \mathrm{aA}$ & 15 & $8,13 \mathrm{aA}$ & 17 & $7,58 \mathrm{cA}$ & 0,47 \\
\hline 13,2 & 14 & $8,00 \mathrm{aA}$ & 13 & $7,09 \mathrm{cA}$ & 26 & $7,57 \mathrm{cA}$ & 0,65 \\
\hline 16,8 & 18 & $8,27 \mathrm{aA}$ & 8 & $7,27 \mathrm{bcA}$ & 20 & $8,33 \mathrm{abA}$ & 0,65 \\
\hline 20,4 & 16 & $7,74 \mathrm{aA}$ & 10 & $8,30 \mathrm{aA}$ & 13 & $7,76 \mathrm{bcA}$ & 0,51 \\
\hline 22,0 & 17 & $8,13 \mathrm{aA}$ & 16 & $8,11 \mathrm{aA}$ & 23 & $8,27 \mathrm{abA}$ & 0,18 \\
\hline DMS & 0,67 & 0,64 & 0,64 & - & & & \\
\hline C.V.(\%) & 10,53 & 10,29 & 9,98 & - & & & \\
\hline
\end{tabular}

${ }^{1}$ Médias seguidas pela mesma letra minúscula, na mesma coluna, e maiúscula, na mesma linha, não diferem significativamente entre si pelo teste de Tukey $(\mathrm{P} \leq 0,05) ;(\mathrm{n})$ : número de insetos usados no teste; Controle 1: Tween $-20(0,1 \%)$; Controle 2: Etanol puro. 
Tabela 3 - Peso de pupas (mg) de A. gemmatalis submetida à aplicação tópica do extrato acetônico das diferentes partes de A. lagesiana (Aristolochiaceae).

\begin{tabular}{llllllll}
\hline Dose $\left(\mu \mathrm{g} . \mu \mathrm{L}^{-1}\right)$ & \multicolumn{7}{c}{ Peso de pupas $(\mathrm{mg})^{1}$} \\
\cline { 2 - 7 } & $(\mathrm{n})$ & Caule/Raiz (aquecido) & $(\mathrm{n})$ & Caule/Raiz (a frio) & $(\mathrm{n})$ & Folha & DMS \\
\hline Controle 1 & 30 & $210,91 \mathrm{aA}$ & 30 & $210,91 \mathrm{aA}$ & 30 & $210,91 \mathrm{aA}$ & - \\
Controle 2 & 30 & $206,64 \mathrm{aA}$ & 30 & $206,64 \mathrm{aA}$ & 30 & $206,64 \mathrm{aA}$ & - \\
6,0 & 26 & $190,27 \mathrm{abA}$ & 25 & $188,89 \mathrm{bcA}$ & 20 & $194,47 \mathrm{abcA}$ & 6,79 \\
9,6 & 22 & $180,66 \mathrm{bA}$ & 22 & $183,07 \mathrm{cA}$ & 19 & $186,19 \mathrm{bcA}$ & 5,94 \\
13,2 & 20 & $174,94 \mathrm{bA}$ & 17 & $193,89 \mathrm{abcA}$ & 27 & $177,20 \mathrm{cB}$ & 11,71 \\
16,8 & 26 & $195,04 \mathrm{abA}$ & 15 & $173,14 \mathrm{cB}$ & 22 & $206,31 \mathrm{abA}$ & 12,03 \\
20,4 & 28 & $175,52 \mathrm{bA}$ & 14 & $184,10 \mathrm{cA}$ & 17 & $183,01 \mathrm{cA}$ & 8,93 \\
22,0 & 20 & $180,87 \mathrm{bA}$ & 21 & $182,32 \mathrm{cA}$ & 28 & $186,12 \mathrm{cA}$ & 6,07 \\
\hline DMS & 23,54 & 20,76 & 22,70 & - & & \\
C.V.(\%) & 15,74 & 13,80 & 14,82 & - & &
\end{tabular}

${ }^{1}$ Médias seguidas pela mesma letra minúscula, na mesma coluna, e maiúscula, na mesma linha, não diferem significativamente entre si pelo teste de Tukey $(\mathrm{P} \leq 0,05) ;(\mathrm{n})$ : número de insetos usados no teste; Controle 1: Tween -20 (0,1\%); Controle 2: Etanol puro.

Tabela 4 - Viabilidade larval (\%) de A.gemmatalis submetida à aplicação tópica do extrato acetônico das diferentes partes de A. lagesiana (Aristolochiaceae).

\begin{tabular}{llclrlrr}
\hline Dose $\left(\mu \mathrm{g} . \mu \mathrm{L}^{-1}\right)$ & \multicolumn{7}{c}{ Viabilidade larval $(\%)^{1}$} \\
\cline { 2 - 7 } & $(\mathrm{n})$ & Caule/Raiz (Aquecido) & $(\mathrm{n})$ & Caule/Raiz (A frio) & $(\mathrm{n})$ & Folha & DMS \\
\hline Controle 1 & 30 & $100,0 \mathrm{aA}$ & 30 & $100,0 \mathrm{aA}$ & 30 & $100,0 \mathrm{aA}$ & - \\
Controle 2 & 30 & $100,0 \mathrm{aA}$ & 30 & $100,0 \mathrm{aA}$ & 30 & $100,0 \mathrm{aA}$ & - \\
6,0 & 30 & $100,0 \mathrm{aA}$ & 23 & $77,0 \mathrm{aA}$ & 22 & $73,0 \mathrm{abA}$ & 28,62 \\
9,6 & 23 & $77,0 \mathrm{aA}$ & 25 & $83,0 \mathrm{aA}$ & 24 & $80,0 \mathrm{abA}$ & 5,76 \\
13,2 & 20 & $67,0 \mathrm{aA}$ & 16 & $53,0 \mathrm{aA}$ & 28 & $93,0 \mathrm{abA}$ & 47,34 \\
16,8 & 28 & $93,0 \mathrm{aA}$ & 27 & $90,0 \mathrm{aA}$ & 18 & $60,0 \mathrm{bA}$ & 35,62 \\
20,4 & 27 & $90,0 \mathrm{aA}$ & 19 & $63,0 \mathrm{aA}$ & 20 & $67,0 \mathrm{abA}$ & 30,78 \\
22,0 & 21 & $70,0 \mathrm{aA}$ & 22 & $73,0 \mathrm{aA}$ & 27 & $90,0 \mathrm{abA}$ & 28,10 \\
\hline DMS & 35,12 & 51,65 & 36,06 & - & & \\
C.V.(\%) & 14,33 & 22,82 & 15,37 & - & & \\
\hline
\end{tabular}

${ }^{1}$ Médias seguidas pela mesma letra minúscula, na mesma coluna, e maiúscula, na mesma linha, não diferem significativamente entre si pelo teste de Tukey $(\mathrm{P} \leq 0,05) ;(\mathrm{n})$ : número de insetos usados no teste; Controle 1: Tween -20 (0,1\%); Controle 2: Etanol puro.

Duração do período pupal - Os valores da duração do período pupal de A.gemmatalis estão descritos na Tabela 2 , verificando-se um valor mais elevado no tratamento "Folha" e na dosagem de $6 \mu \mathrm{g} /$ inseto $(8,45$ dias), sendo este valor semelhante ao da testemunha (8,25 dias). Foi observado também que nos tratamentos "Caule/Raiz (a frio)" e "Folha", na dosagem de $13,2 \mu \mathrm{g} /$ inseto, houve uma redução deste parâmetro, sendo de 7,09 e 7,57 dias, respectivamente. Esta redução pode ser explicada pelo fato de que o inseto, possivelmente, tenha acelerado este período para tentar chegar mais rápido à fase adulta e, consequentemente, gerar descendentes.
Peso pupas - Observando-se o peso de pupas de A. gemmatalis (Tabela 3), notou-se que os diferentes tratamentos, de modo geral, influenciaram significativamente na sua redução sendo os menores valores obtidos nos tratamentos "Caule/Raiz (aquecido)" e "Folha", na dosagem de 13,2 $\mu \mathrm{g} /$ inseto e "Caule/ Raiz (a frio)", na dosagem de 16,8 $\mu \mathrm{g} /$ inseto, respectivamente de 174,$94 ; 177,20$ e 173,14 mg. Assim, o efeito adverso dos extratos em relação ao inseto foi observado, pois o peso de pupas está relacionado diretamente como potencial biótico desta espécie. Estes resultados corroboram também os dados de Roel (2001). 
Tabela 5 - Viabilidade pupal (\%) de A. gemmatalis submetida à aplicação tópica do extrato acetônico das diferentes partes de A. lagesiana (Aristolochiaceae).

\begin{tabular}{|c|c|c|c|c|c|c|c|}
\hline \multirow[t]{2}{*}{ Dosagem $\left(\mu \mathrm{g} \cdot \mu \mathrm{L}^{-1}\right)$} & \multicolumn{7}{|c|}{ Viabilidade pupal $(\%)^{1}$} \\
\hline & (n) & Caule/Raiz (Aquecido) & (n) & Caule/Raiz (A frio) & (n) & Folha & DMS \\
\hline Controle 1 & 28 & $90,0 \mathrm{aA}$ & 28 & $90,0 \mathrm{aA}$ & 28 & $90,0 \mathrm{aA}$ & - \\
\hline Controle 2 & 28 & $90,0 \mathrm{aA}$ & 28 & $90,0 \mathrm{aA}$ & 28 & $90,0 \mathrm{aA}$ & - \\
\hline 6,0 & 26 & $77,0 \mathrm{aA}$ & 25 & $83,0 \mathrm{aA}$ & 20 & $94,0 \mathrm{aA}$ & 39,53 \\
\hline 9,6 & 22 & $72,0 \mathrm{aA}$ & 22 & $70,0 \mathrm{aA}$ & 19 & $90,0 \mathrm{aA}$ & 38,17 \\
\hline 13,2 & 20 & $67,0 \mathrm{aA}$ & 17 & $74,0 \mathrm{aA}$ & 27 & $95,0 \mathrm{aA}$ & 42,09 \\
\hline 16,8 & 26 & $70,0 \mathrm{aA}$ & 15 & $69,0 \mathrm{aA}$ & 22 & $92,0 \mathrm{aA}$ & 38,25 \\
\hline 20,4 & 28 & $57,0 \mathrm{aA}$ & 14 & $71,0 \mathrm{aA}$ & 17 & $81,0 \mathrm{aA}$ & 35,18 \\
\hline 22,0 & 20 & $83,0 \mathrm{aA}$ & 21 & $78,0 \mathrm{aA}$ & 28 & $82,0 \mathrm{aA}$ & 24,32 \\
\hline$\overline{\mathrm{DMS}}$ & 50,28 & 56,02 & 31,25 & - & & & \\
\hline C.V.(\%) & 23,46 & 25,39 & 12,41 & - & & & \\
\hline
\end{tabular}

${ }^{1}$ Médias seguidas pela mesma letra minúscula, na mesma coluna, e maiúscula, na mesma linha, não diferem significativamente entre si pelo teste de Tukey ( $\left.\mathrm{P} \mathrm{d}^{\prime \prime} 0,05\right)$; (n): número de insetos usados no teste; Controle 1: Tween $-20(0,1 \%)$; Controle 2: Etanol puro.

Viabilidade larval - Pelos resultados apresentados para viabilidade larval de A. gemmatalis (Tabela 4) verificou-se que os maiores índices de mortalidade de lagartas ocorreram nos tratamentos "Caule/ Raiz (aquecido)" e "Caule/Raiz (a frio)", na dosagem de 13,2 $\mu \mathrm{g}$ /inseto e "Folha", na dosagem de 16,8 $\mu \mathrm{g} /$ inseto, sendo, respectivamente, 67, 53 e $60 \%$. Embora os tratamentos "Caule/Raiz (aquecido)" e "Caule/ Raiz (a frio)" na dose de 13,2 $\mu \mathrm{g} /$ inseto, e "Caule/ Raiz (a frio)" e "Folha" na dose de 20,4 gg/inseto não tenham diferido dos controles (onde a viabilidade foi de $100 \%$ ), os valores obtidos foram menores que $70 \%$. NASCIMENTO et al. (2003) também verificaram que a viabilidade larval de A. gemmatalis foi afetada negativamente pela pulverização de substâncias puras isoladas dos extratos acetônico e etanólico de raízes de A. pubescens.

Viabilidade pupal - Observando os dados relativos à viabilidade pupal de A. gemmatalis nos diferentes tratamentos (Tabela 5), verificou-seque não houve diferença estatística entre os valores. Contudo, se for calculada a viabilidade total dos indivíduos que chegaram à fase adulta, por exemplo, para a dosagem de $20,4 \mu \mathrm{g}$ /inseto, conclui-se que menos de $50 \%$ dos insetos completaram seu ciclo de vida, portanto, neste tratamento houve um controle de mais da metade do número de insetos inicial.

\section{CONCLUSÕES}

Nas condições em que os experimentos foram conduzidos, foi possível concluir que o extrato acetônico das diferentes partes de A. lagesiana afetam a biologia da lagarta-da-soja, A. gemmatalis, pois, dependendo da dose e da parte da planta utilizada para extração, ocorre alongamento do período larval e redução do peso de pupas e da viabilidade larval.

\section{AGRADECIMENTOS}

À Coordenação de Aperfeiçoamento de Pessoal de Nível Superior (CAPES), pela concessão de bolsa ao primeiro autor. À Estação Experimental Agrícola da Du Pont do Brasil S/ A, pelo constante apoio à pesquisa.

\section{REFERÊNCIAS}

CAMPO, C.B.H.; OLIVEIRA, E.B. de; MOSCARDI, F. Criação massal da lagarta da soja (Anticarsia gemmatalis). Londrina: EMBRAPA - CNPSO, 1985. 23p. (Documentos).

CICCIA, G.; COUSSIO, J.; MONGELLI, E. Insecticidal activity against Aedes aegypti larvae of some medicinal south american plants. Journal of Etnopharmacology, v.72, n.1/2, p.185-189, 2000.

GAZZONI, D.L.; OLIVEIRA, E.B. de; CORSO, I.C.; FERREIRA, B.S.C.; VILLAS BÔAS, G.L.; MOSCARDI, F.; PANIZZI, A.R. Manejo de pragas da soja. Londrina: EMBRAPA-CNPSo, 1981. 44p. (Circular Técnica).

GRAINGE, M.; AHMED, S. (Ed.). Handbook of plants with pestcontrol properties. New York: John Wiley, 1988. 400p.

LOPES, L.M.X.; NASCIMENTO, I.R., SILVA, T.

Phytochemistry of the Aristolochiaceae family. Global Research Network, v.2, p.19-108, 2001. 
MARANHÃO, Z.C. Plantas inseticidas. Revista da Agricultura, v.29, n.3/4, p.113-121, 1954.

NASCIMENTO, I.R.; MURATA, A.T.; BORTOLI, S.A.; LOPES, L.M.X. Insecticidal activity of chemical constituents from Aristolochia pubescens against Anticarsia gemmatalis larvae. Pest Management Science, v.60, n.1, p.413-416, 2003.

ROEL, A.R. Utilização de plantas com propriedades inseticidas: uma contribuição para o desenvolvimento rural sustentável. Revista Internacional de Desenvolvimento, v.1, n.2, p.43-50, 2001.

SCHMUTTERER, H. Potential of azadirachtin containing pesticides for integrated pest control in developing and industrialized countries. Journal of Insect Physiology, v.34, n.7, p.713-719, 1988.

SECOY, D.M.; SMITH, A.E. Use of plants in control of agricultural and domestic pests. Economic Botany, v.37, n.1, p.28-57, 1983.

SIME, K.R.; FEENY, P.P.; HARIBAL, M.M.

Sequestration of aristolochic acids by the pipevine swallowtail, Battus philenor (L.): evidence and ecological implications. Chemoecology, v.10, n.4, p.169$178,2000$.
VENDRAMIM, J.D.; CASTIGLIONI, E. Aleloquímicos, resistência de plantas e plantas inseticidas. In: GUEDES, J.C.; COSTA, I.D. da; CASTIGLIONI, E. (Org.). Bases e técnicas do manejo de insetos. Santa Maria: UFSM/ CCR/DFS, 2000. p.113-128.

VENKADASUBRAMANIAN, V.; DAVID, P.M.M. Insecticidal toxicity of commercial Bacillus thuringiensis (Berliner) products in combination with botanical to Spodoptera litura (Fabricius) and Helicoverpa armigera (Hüebner). Journal of Biological Control, v.13, p.85-92, 1999.

WU, T.S.; LEU, Y.L.; CHAN, Y.Y. Aristolochic acids as a defensive substance for the Aristolochiaceous plantfeeding swallowtail butterfly, Pachliopta aristolochiae interpositus. Journal of the Chinese Chemical Society, v.47, n.1, p.221-226, 2000.

ZARROUG, J.M.A.; NAGGUD, A.D.; BASHIN, A.D.; MAGEED, A.A. Evaluation of Sudanese plants. International Journal of Crude Drugs Research, v.26, n.2, p.7-80, 1988.

Recebido em 19/4/07

Aceito em 20/2/09 